\title{
UNSUPERVISED VOCABULARY DISCOVERY USING NON-NEGATIVE MATRIX FACTORIZATION WITH GRAPH REGULARIZATION
}

\author{
Meng Sun, Hugo Van hamme \\ Department of Electrical Engineering-ESAT, Katholieke Universiteit Leuven, \\ Kasteelpark Arenberg 10, Bus 2441, B-3001 Leuven, Belgium \\ mengsun@esat.kuleuven.be, hugo.vanhammedesat.kuleuven.be
}

\begin{abstract}
In this paper, we present a model for unsupervised pattern discovery using non-negative matrix factorization (NMF) with graph regularization. Though the regularization can be applied to many applications, we illustrate its effectiveness in a task of vocabulary acquisition in which a spoken utterance is represented by its histogram of the acoustic co-occurrences. The regularization expresses that temporally close co-occurrences should tend to end up in the same learned pattern. A novel algorithm that converges to a local optimum of the regularized cost function is proposed. Our experiments show that the graph regularized NMF model always performs better than the primary NMF model on the task of unsupervised acquisition of a small vocabulary.
\end{abstract}

Index Terms - Vocabulary discovery, Non-negative matrix factorization, Graph regularization, Spectral clustering

\section{INTRODUCTION}

Unsupervised learning aims at discovering objects or concepts from input information without human effort. In spoken pattern discovery, it has at least two advantages against the supervised learning. Firstly, unsupervised learning is not affected by the assumptions and limitations of language theories. Statistical pattern recognition may be better accomplished by a system which is to model the purposeful processing of speech for communication, instead of being designed to emulate theories about the structure of speech and language [1]. The purposeful processing can have different layers to make hierarchical abstraction. At each level, we don't know the "right" representation, so local features can be extracted in an unsupervised way [2]. Secondly, unsupervised learning doesn't need a huge set of hand-labeled examples. Also, for both supervised and unsupervised learning, one may encounter new words (out of vocabulary) that need to be detected, learned and added to the vocabulary unsupervisedly.

Unsupervised sequential pattern discovery can be based on the trace or path search and matching [3][4][5]. Short re-

The research was funded by the K.U.Leuven research gran OT/09/028(VASI) curring traces are segmented and extracted by dynamic time warping (DTW). The process of vocabulary learning is then mimicked using the obtained short spoken traces and grounding truth information [3][5]. In a completely unsupervised way, the similarities between the traces are used as criteria to clustering to obtain word-like patterns [4]. The process of generating sequential patterns is straightforward and plausible, but it doesn't escape the time-demanding DTW process. However, the fairly strict pattern matching in DTW may not be a must to speech recognition and classification, so other representation techniques with lower complexity that nonetheless retain sufficient information for the recognition and classification are worth exploring.

In this paper, we use a recently proposed representation of speech, histograms of the acoustic co-occurrence (HAC) [6], a non-negative representation of speech in which the HAC of an utterance is approximately the weighted sum of the HAC of its compounding words. Hence, the HAC of words can be found with non-negative matrix factorization (NMF) as the additive parts that make up a collection of utterance-level HACs. While several choices can be made for the acoustic events in HAC (e.g. DTW templates, phones or VQ-based spectral matches), this paper will be based on the posterior probabilities of prototypical Gaussians matching short-term speech spectra via the Mel-frequency cepstral coefficients (MFCC) representation. However, the co-occurrence probabilities finally used in the HAC representation are only a weak representation of temporal order. With supervision as in [6], the loss of temporal order is not so harmful to the recognition accuracy as the supervision can help to group the co-occurrences belonging to a vocabulary pattern together. But if without supervision, the model can't ensure that the patterns correspond to HACs of traces that make sense in time, which can lead to inaccurate solutions.

In order to constrain the NMF solutions, graph regularization is applied for each pattern, itself a HAC representation, to favor that temporally adjacent co-occurrences that are probably part of the same word can also stay together in an obtained pattern. Hence, with graph regularization, we prefer patterns that have high values for co-occurrences that have been ob- 
served closely in time. The idea of graph regularization was also proposed in [7] where it was used to preserve the geometric structure of the data on a manifold. However, our goal is different, which leads to a different objective function and updating algorithms.

This paper is organized as follows: in section 2, the NMF model and algorithm with graph regularization are described. The results and discussion are in section 3 and section 4 . The conclusion is in section 5 .

\section{NMF MODEL WITH GRAPH REGULARIZATION FOR VOCABULARY DISCOVERY}

NMF was originally proposed to learn parts from images in [8]. It performs well at finding recurring patterns from multiple samples of a pattern mixture. In this paper, the patterns that describe spoken vocabularies are described by a HACrepresentation. They are obtained as a non-negative matrix factor of a data matrix $V$ in which each column is a HAC representation of an utterance [6].

\subsection{The HAC-NMF model for speech recognition}

The procedure to get the HAC representation is as follows.

\section{- Gaussians and Posterior Probabilities} Firstly, the utterance is chopped up in overlapping signal analysis frames. For each frame, a MFCC vector $x$ is computed. A number of Gaussians $\mathcal{N}\left(\mu_{i}, \Sigma_{i}\right), 1 \leq$ $i \leq M$ are then trained unsupervisedly using the EMalgorithm. The likelihood of all Gaussians $p\left(x ; \mu_{i}, \Sigma_{i}\right)$ is evaluated for each frame and the top- $K$ probabilities are retained and normalized to sum to unity to yield a sparse multinomial probability distribution $\left\{\hat{p}\left(x ; \mu_{i}, \Sigma_{i}\right)\right\}$.

\section{- Co-occurrence Statistics}

The feature vectors $x_{t_{1}}$ and $x_{t_{2}}$ are said to form a frame-pair for a given lag-value if lag $=t_{2}-t_{1}$. For each frame-pair, $K \times K$ Gaussian-pairs among $\left\{\hat{p}\left(x_{t_{1}} ; \mu_{i}, \Sigma_{i}\right) \times \hat{p}\left(x_{t_{2}} ; \mu_{j}, \Sigma_{j}\right) \mid i, j=1, \ldots, M\right\}$ will have positive activation scores. For a given lag-value, the $(i \times(M-1)+j)$-th element of the $M^{2}$-dimensional HAC representation of an utterance is obtained by accumulating the activation scores of the Gaussian-pairs over all frame-pairs of an utterance, $\sum_{t_{2}-t_{1}=l a g} \hat{p}\left(x_{t_{1}} ; \mu_{i}, \Sigma_{i}\right) \times \hat{p}\left(x_{t_{2}} ; \mu_{j}, \Sigma_{j}\right)$, which forms a column of the data matrix $V_{\text {lag }}$. We can take several different lags to capture rich context dependency information and stack the data matrices to form $\left[V_{l a g 1} ; V_{l a g 2} ; V_{l a g 3}\right]$.

For the NMF model, in the training stage, by solving $\min _{W, H} C_{0}(V \| W H)$ where $C_{0}$ is the extended KullbackLeibler divergence [6], we can get the vocabulary patterns as the columns of $W$ and the activations of the patterns as the columns of $H$. The lack of supervisory data makes it however difficult to interpret the discovered patterns in $W$. For evaluation purposes, we estimate a mapping matrix $Q$ that maps the activations to the ground truth by solving $\min _{Q} C_{0}(G \| Q H)$, where $G$ is constructed as follows: if the $n$-th utterance is known to contain the $m$-th vocabulary item $l$ times, $G_{m n}=l$; otherwise, $G_{m n}=0$.

In the testing stage, we firstly estimate the activations $H^{\prime}$ of the patterns $W$ by $\min _{H^{\prime}} C_{0}\left(V^{\prime} \| W H^{\prime}\right)$ on the testset. Subsequently, the activations of the vocabulary items is computed as $\hat{G}^{\prime}=Q H^{\prime}$ using the mapping matrix $Q$ derived from the training data. Since $\hat{G}^{\prime}$ indicates the presence of the learned vocabulary items in each test utterance without ordering them in time, the performance metric that is adopted here is an unordered error rate. Suppose that the number $D_{u}$ of different digits occurring in the $u$-th test utterance is given, the $D_{u}$ candidates with highest activation are retained in the $u$-th column of $\hat{G}^{\prime}$. The word error rate is then defined as the sum of the number of incorrect digits (insertion, deletion), divided by the sum of $D_{u}$ over the complete test set [9].

\subsection{Graph regularization}

In the above unsupervised NMF model, there is no constraint that the columns of $W$ must correspond to HACs of traces that make sense in time, which may lead to unrealistic solutions. The role of the graph regularization is to make sure the adjacent (see below) Gaussian-pairs have nearly the same activations in a pattern $W_{k}$ (the $k$-th column of $W$ ). Suppose we know the symmetric adjacency matrix $U$ of the Gaussianpairs and represent them as the undirected graph Laplacian $L=D-U$, where $D$ is the degree matrix. Define the function of the graph constraint as in Equation (1) [10].

$$
W_{k}^{T} L W_{k}=\sum_{u \sim v}\left(W_{u k}-W_{v k}\right)^{2} U_{u v}
$$

where $\sum_{u \sim v}$ denotes the sum over all unordered Gaussianpairs $u, v$ for which $u$ and $v$ are adjacent in $U$.

Now we present how to build the undirected graph of the Gaussian-pairs. Firstly, we define the adjacency of framepairs. We suppose that the temporal property of a framepair is given by its head frame, i.e. whether two frame pairs are adjacent or not is determined by their first frame. If the two heads are spaced less than pNear frames, we say the two frame-pairs are adjacent. Two Gaussian-pairs are adjacent if they appear in the Nbest $\left(\leq K^{2}\right)$ list of Gaussian-pairs of two adjacent frame-pairs. The total adjacency frequency of two Gaussian-pairs is the accumulation over all the adjacent frame-pairs of the utterances of the training set.

For the HACs with $l a g_{i}$ and $l a g_{j}$, we can get one adjacency matrix $U^{(i j)}$. Hence, for three lags, the final adjacency 
matrix $U$ would be Equation 2.

$$
U=\left[\begin{array}{lll}
U^{(11)} & U^{(12)} & U^{(13)} \\
U^{(21)} & U^{(22)} & U^{(23)} \\
U^{(31)} & U^{(32)} & U^{(33)}
\end{array}\right]
$$

Because the adjacency matrix is made from data which contains a lot of uncertainty or variation of speech, not all of the connected Gaussian-pairs in the obtained adjacency matrix are really neighbors in the expected ideal patterns. In order to remove spurious adjacency relations and to make the model robust, a threshold is applied to make $U$ logical.

The optimization problem becomes

$$
C(V \| W, H)=C_{0}(V \| W, H)+\lambda C_{1}(W)
$$

where, $C_{1}(W)=\operatorname{Tr}\left(W^{T} L W\right), \lambda$ is a parameter to balance the two terms: divergence and regularization.

\subsection{Updating algorithm with $L_{1}$ normalization}

Each pattern is modeled as a multinomial distribution of the activations on the \#lags $\times M \times M$ Gaussian-pairs, where \#lags is the number of lags. Hence for any column of $W,\left\|W_{k}\right\|_{1}=$ 1. The optimization over $W_{k}$ subject to this linear constraint is the solution of Equation (4).

$$
\begin{aligned}
2 \lambda D_{i i} W_{i k}^{2}+\sum_{s}\left(B_{s k}+2 \lambda E_{s k}\right) W_{i k} & \\
-2 \lambda \sum_{s} D_{s s} W_{s k}^{2} W_{i k}-B_{i k}-2 \lambda E_{i k} & =0
\end{aligned}
$$

where $B_{i k}=\sum_{j} V_{i j} \frac{W_{i k}^{t} H_{k j}}{\sum_{t, b} W_{i b}^{t} H_{b t}}, C_{k}=\sum_{j} H_{k j}, E_{i k}=$ $\left(U W^{t}\right)_{i k} W_{i k}^{t}$.

We were unable to find an analytical solution for each $W_{i k}$. Instead we construct a contraction mapping in Equation (5) to solve the equations iteratively.

$$
W_{i k}^{(n+1)}=\frac{-b_{i k}^{(n)}+\sqrt{\left(b_{i k}^{(n)}\right)^{2}+4 a_{i} c_{i k}}}{2 a_{i}}
$$

where $a_{i}=2 \lambda D_{i i}, b_{i k}^{(n)}=\sum_{s} B_{s k}-2 \lambda \sum_{s} D_{s s}\left(W_{s k}^{(n)}\right)^{2}+$ $2 \lambda \sum_{s} E_{s k}, c_{i k}=B_{i k}+2 \lambda E_{i k}>0$. For the rows $i$ with positive degrees in $D$, we have $W_{i k}^{(n+1)}>0$. However, we should notice that, due to the sparsity of the co-occurrence features and by making the adjacency matrix $U$ logical, many vertices (Gaussian-pairs) in the graph end up isolated, i.e. with zero degree. Their updating formula is in Equation (6). We can see that $\lambda=0$ just corresponds the normal NMF without the graph regularization.

$$
W_{i k}^{(n+1)}=\frac{c_{i k}}{b_{i k}}
$$

If Equation (5) is a contraction mapping, its limits are the solutions of Equation (4). We can prove that the contraction mapping and the $L_{1}$ normalization of columns of $W$ also hold when $\sum_{s \in I} B_{s k}-2 \lambda \sum_{s} D_{s s} W_{s k}^{2}+2 \lambda \sum_{s} E_{s k}>0$. It is interesting to note that this condition also ensures that Equations (6) with zero graph degrees have positive roots. In our numerical experiments, the contraction mapping converges quickly with at most 5 iterations. Due to space limitation, we leave the complete proof for a later publication.

\section{RESULTS OF VOCABULARY DISCOVERY}

The experiments were made on the TI-DIGITS database which contains 11 digits of the 6026 training utterances (each with at least two digits) and 1001 test utterances from male and female speakers. The window length for spectral analysis was $20 \mathrm{~ms}$ and the frame shift was $10 \mathrm{~ms}$. The MFCC extraction used 30 Mel-filter banks from which 12 MFCC coefficients are computed plus the frame's log-energy. The three vectors of Static, Velocity, Acceleration were concatenated to a 39-dimensional feature vector on which a Gaussian mixture of $M=200$ components were trained unsupervisedly on the training set. For each frame, at most $K=3$ Gaussians were retained. The lags of the frame-pairs were 20, 50 and $90 \mathrm{~ms}$, which represent contextual dependence with different time scales. For the graph model, the top Nbest=4 Gaussianpairs were retained for each frame-pair, and the neighborhood parameter is $p$ Near $=3$ frames $(30 \mathrm{~ms})$.

A reasonable value for the parameter threshold to make the adjacency matrix logical is obtained as follows. Suppose different patterns have different Gaussian-pairs. The minimum frequency of adjacency of a Gaussian-pair approximates the average frequency of the patterns in the training dataset. Treating digits from male and female speakers as different patterns, the average frequency of a pattern is 6026 (\# utterances)/11 (\# digits)/2 (\# genders) $\approx 275$. Since overestimating the threshold leads to the standard NMF, we conservatively take a slightly larger threshold $=300$.

Too big $\lambda$ will over-regularize the columns of $W$, while too small ones may not perform the regularization effectively. The role of the graph regularization should be assistant and not be dominating the learning problem. So we control that $\lambda C_{1}$ (end) $\leq \frac{1}{10} C_{0}$ (end). For the experiments in this paper, we took $\lambda=100$ and $\lambda=10$ to test the parameter sensitivity.

Figure 1 shows the change of word error rates w.r.t. the factorization dimensions $R$ and the comparison among different models. The range of each error bar is the standard deviation of the mean value over 5 decomposition attempts, treating each as a Bernoulli trial. 500 iterations were made for each parameter setup. For the supervised baseline, we made the factorization $[G ; V]=[Q ; W] H$ to estimate $Q$ and $W$ as in [6] and evaluated the performance as in Section 2.

\section{DISCUSSION}

By enlarging the dimension from $R=20$ to $R=60$, we observe an improvement for all the models: for the supervised 


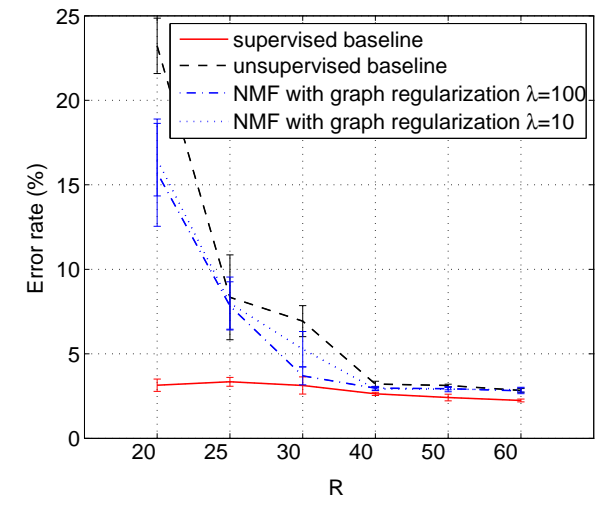

Fig. 1. Comparison of word error rates among supervised, unsupervised and graph regularized NMF models.

NMF, from $3.14 \%$ to $2.24 \%$; for the unsupervised NMF, from $23.22 \%$ to $2.84 \%$; for the NMF with graph regularization, from $15.72 \%$ to $2.82 \%$ with $\lambda=100$, from $16.49 \%$ to $2.88 \%$ with $\lambda=10$. The steepest descent happens from $R=20$ to $R=25$ in the unsupervised models, which indicates the inherent dimension of the data may be between 20 and 25. By checking the mapping matrix $Q_{11 \times R}$ and the activation matrix $H_{R \times 1001}^{\prime}$ on the testset, we found that different patterns associated with the same digit are activated by either male speech or female speech. That is the models treated male and female digits as different patterns automatically, so the dimension of the data is 22 which is indeed between 20 and 25 . Like in an Artificial Neural Network (ANN), the number of nodes should be the minimal number that is required to solve a problem. $R \leq 22$ is not sufficient to obtain a reasonable solution, while for $R \geq 30$ the model complexity is high enough and the accuracy levels off. Actually, the performance w.r.t. $R$ is also highly related with the structure of the data and the features used in the model.

The accuracy of the supervised model is fairly independent of the number of patterns $(R)$, because the supervisory information structures the co-occurrences in the relevant patterns. The graph regularized model seems to successfully group the co-occurrence patterns when the number of patterns is large enough $(R=30)$, as its accuracy is closer to the performance of the supervised model than the accuracy of the NMF model without regularization. When $R$ is overestimated $(R \geq 40)$, both the regularized and the unregularized model discover useful patterns. In this case of small vocabulary, these are sufficient to maintain the accuracy.

It is worth noting that computationally, graph regularization is well-behaved. Building the adjacency matrix is linear in the amount of training data and involves storing cooccurrence counts, much like what is required in building bigram language models. The most expensive part is still computing $W H$, like in the unregularized problem.

\section{CONCLUSION}

We presented an NMF model with graph regularization to unsupervised digit pattern discovery. The representation of each spoken digit was based on its histogram of acoustic cooccurrences (HAC) which was chosen to be the co-occurrence probability of Gaussian-pairs. Graph regularization was used to retain the temporal closeness of the Gaussian-pairs. A new algorithm was applied to get a local optimum of the optimization problem. The algorithm ensures each obtained pattern is a multinomial distribution of the activations of the Gaussianpairs. The experiments showed that the graph regularized NMF model always outperforms the primary NMF model on the unsupervised vocabulary acquisition on TI-DIGITS database. The model can be useful to extract patterns of the unsupervised low levels of a deep learning model by preserving the structural information of the features.

\section{REFERENCES}

[1] L.Boves, L.ten Bosch, R.Moore, "ACORNS-towards computational modeling of communication and recognition skills", Proc. ICCI 2007.

[2] Y.Bengio, "Learning deep architectures for AI", Foundations and Trends in Machine Learning, vol.2, no.1, pp.1-127, 2009.

[3] L.ten Bosch, B.Cranen, "A computational model for unsupervised word discovery", Proc. Interspeech 2007, pp.1481-1484.

[4] A.Park, J.R.Glass, "Unsupervised Pattern Discovery in Speech", IEEE Transactions on Audio, Speech and Language Processing, vol.16, no.1, pp.186-197, 2008.

[5] G.Aimetti, L.ten Bosch, R.K.Moore, "The Emergence of Words: Modelling Early Language Acquisition with a Dynamic Systems Perspective”, Proc. Interspeech 2009.

[6] H.Van hamme, "HAC-models: a Novel Approach to Continuous Speech Recognition", Proc. Interspeech 2008, pp.2554-2557.

[7] D.Cai, X.He, X.Wu,J.Han, "Non-negative Matrix Factorization on Manifold”, Proc. ICDM 2008, pp.63-72.

[8] D.D.Lee, H.S.Seung, "Learning the parts of objects by non-negative matrix factorization", Nature 401, pp.788$791,1999$.

[9] M.Van Segbroeck, H.Van hamme, "Unsupervised learning of time-frequency patches as a noise-robust representation of speech", Speech Communication, vol.51, no.11, pp.1124-1138, November 2009.

[10] F.Chung, "Spectral Graph Theory", CBMS Regional Conference Series in Mathematics, no.92, 1997. 